Die ebenfalls von der KVTG entwickelte „eNachricht 2.0“ wird hingegen bislang nur von wenigen Softwarehäusern unterstützt. Sie entspricht einer E-Mail-Nachricht, über die Praxen - mit anderen Ärzten und KVen - über den sicheren KV-Connect-Kanal Informationen samt Dateianhängen austauschen können. Das Ganze läuft ebenfalls direkt aus dem Primärarztsystem heraus. Laut dem Audit-Register der KVTG wurden bislang 20 Systeme dafür erfolgreich auditiert - in zwei Fällen lief die Auditierung über den KV-SafeNet-Router GUSbox der Deutschen Gesundheitsnetz Service $\mathrm{GmbH}$ (DGN).

Das liegt aber auch daran, dass viele Softwarehäuser eigene sichere Kommunikationswege bieten. Bei tomedo gibt es laut Johannes Zollmann verschiedene Möglichkeiten, um unter tomedo-Praxen Daten direkt auszutauschen. Die CompuGroup hat den sicheren Kommunikationskanal „CGM Connect“ im Angebot, über den unter CGM-Kunden elektronische Briefe laufen und der auch als Chatfunktion genutzt werden könne. Außer- dem stünden über die CGM LIFE-Akte des Patienten zusätzliche Möglichkeiten eines sicheren Datenaustausches zur Verfügung.

Epikur arbeitet laut Marcus Krüger, Bereichsleiter Marketing beim gleichnamigen Softwarehaus, zudem mit dem eBrief der PIN Mail AG. „Der eBrief verbindet moderne Technologie mit den Vorteilen der klassischen Kommunikation“, erläutert Krüger. „Der Behandler lädt seine Dokumente elektronisch hoch. Die Dokumente werden von der PIN Mail AG gedruckt, kuvertiert, frankiert und in die Zustellung übergeben." Alle Verbindungen für die Datenübertragung seien nach höchsten Sicherheitsstandards verschlüsselt und alle Mitarbeiter im Verarbeitungsprozess unterlägen sowohl dem Bundesdatenschutzgesetz als auch dem Postgeheimnis, versichert er.

Da die Kommunikation mit dem Patienten immer wichtiger wird, stehen auch hierfür Möglichkeiten bereit: Bei Epikur ist dies etwa der SMS-Versand aus dem System. Medatixx hat hierfür mit der $\mathrm{Pa}-$ tientenservice-App x.patient einen eige- nen Kanal entwickelt, über den eine sichere Verbindung zur Praxissoftware aufgebaut wird und dann direkt aus den Medatixx-Systemen heraus Daten mit $\mathrm{Pa}$ tienten ausgetauscht werden können. $\mathrm{Na}$ türlich lassen sich auch externe E-MailDienste einbinden - die meisten Softwarehäuser können einen Zugriff etwa zu Microsofts Outlook oder Mozillas Thunderbird direkt über ihr System ermöglichen.

Ob die Daten dann allerdings sicher übertragen werden, hängt am E-MailProvider. Indamed empfiehlt Praxen, hier die sicherere TLS-Verschlüsselung zu nutzen, weil hier das Versandprotokoll in sechs Teile aufgesplittet werde. Um eine E-Mail lesen zu können, würden alle sechs Teile (für die Technikaffinen: Record-, Handshake-, Change-, Cipher-, Spec- und Alert-Protokoll) in der richtigen Reihenfolge benötigt, was potenzielle Angriffe erschwere. Die Verschlüsselung lässt sich laut Indamed dann ebenfalls direkt aus der Praxissoftware heraus anstoßen.

RebekkaHöh

Health-Apps

\title{
Ein Siegel aus KV-Hand
}

Die KV Telematik GmbH will ab 2018 zur Anlaufstelle im

App-Dschungel werden - für Gründer aber auch für Ärzte.

Balsam für die Ärzte: Bremser in Sachen Digitalisierung sind die niedergelassenen Ärzte laut Dr. Florian Fuhrmann, Geschäftsführer der KV Telematik $\mathrm{GmbH}$, nicht. Ganz im Gegenteil: In den Praxen habe die Digitalisierung bereits vor über 30 Jahren mit der Einführung der Desktop-PC angefangen, erläuterte er auf dem gevkoSymposium „Digitales Gesundheitswesen 2021“ in Berlin. „Genau diese frühe Digitalisierung im Gesundheitswesen wird nun zum Boomerang." Denn die Transformation in die mobile Welt sei im Versorgungsalltag schwierig.

Genau hier will nun die KV Telematik GmbH (KVTG) ansetzen. Denn bis zu einer gewissen technischen Sättigung hätten die Praxen gut mitgemacht. Ab 2018 will die KBV-Tochter Orientierung im un- durchsichtigen Health-App-Markt bieten. Dazu soll, wie Fuhrmann berichtete, ein systematisches Markt-Screening stattfinden.

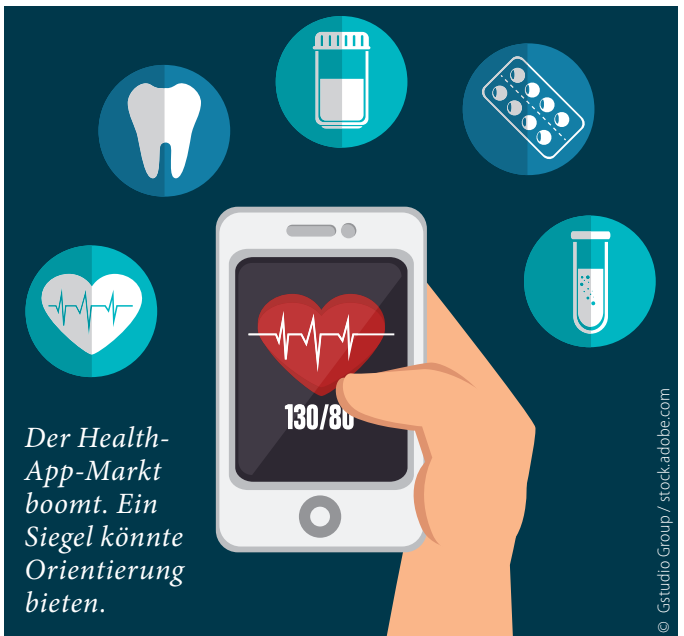

Gemeinsam mit medizinischen Fachgesellschaften wolle man ein inhaltliches Siegel für Health-Apps entwickeln. Gleichzeitig sieht sich die KVTG als Anlaufstelle für Unternehmen, Investoren und KVen, die neue Ideen in diesem Segment entwickeln wollen. Fuhrmann:

„Wir wollen das Gesundheitswesen erläutern und Impulse setzen." Allerdings will die KVTG hier nicht als Geldgeber fungieren. „Wir wollen als Art Incubator ein Umfeld schaffen, das den Unternehmen hilft, sich im Gesundheitsmarkt zurechtzufinden."

Eher bekannt für Vernetzungsanwendungen wie den E-Arztbrief oder den Kommunikationskanal KV-Connect, will die KBV-Tochter Fuhrmann zufolge künftig aber auch eigene KV-Apps entwickeln. An erster Stelle steht dabei eine Notfall-App, die auch den Weg in die Telematikinfrastruktur finden soll.

Rebekka Höh 